\title{
Cuidados Paliativos em Pacientes com Câncer Avançado e Covid-19
}

doi: https://doi.org/10.32635/2176-9745.RBC.2020v66nTemaAtual.1077

\author{
Palliative Care for Patients with Advanced Cancer and COVID-19 \\ Cuidados Paliativos a Pacientes con Cáncer Avanzado e Covid-19
}

\begin{abstract}
Renata de Freitas ${ }^{1}$; Luciana Aparecida Faria de Oliveira ${ }^{2}$; Karla Santos da Costa Rosa ${ }^{3}$; Alessandra Zanei Borsatto4; Simone Garruth dos Santos Machado Sampaio ${ }^{5}$; Bianca Ribeiro Sales ${ }^{6}$; Mabel Viana Krieger7; Eliza Maffioletti Furtunato Leocádio Esteves ${ }^{8}$; Eliana David da Silva'; Livia Costa de Oliveira ${ }^{10}$
\end{abstract}

\section{INTRODUÇÃO}

Encontramo-nos em estado de pandemia pela doença pelo coronavírus 2019 (do inglês, coronavirus disease 2019 - Covid-19), declarada pela Organização Mundial da Saúde (OMS) em 11 de março de 2020. O vírus causador da doença pertence à família Coronaviridae e foi denominado Sars-CoV-2 (do inglês, severe acute respiratory syndrome coronavirus 2). A doença já acumula milhares de indivíduos infectados, falecidos e curados em todo o mundo ${ }^{1}$. O conhecimento científico sobre a epidemiologia e o amplo espectro clínico da Covid-19 ainda não estão descritos completamente, também como, ainda, não se sabe com clareza seu padrão de letalidade, mortalidade, infectividade e transmissibilidade. Entre os fatores de risco identificados para ocorrência de quadros clínicos graves, estão a idade avançada e a presença de comorbidades, como doenças cardiovasculares, diabetes, doença respiratória crônica, doenças imunossupressoras, câncer, entre outras² .

Tratando-se o câncer de uma doença crônica com necessidade de tratamentos agressivos, os pacientes oncológicos compóem um grupo mais suscetível a infecçôes como a Covid-193. Conforme ressaltado por Thuler e Melo ${ }^{4}$, portadores de neoplasia maligna possuem, provavelmente, maior risco de desenvolver formas mais graves da infecção.

No contexto da atenção ao paciente com doença oncológica, acometido ou não pela Covid-19, a linha de cuidado deve contemplar os cuidados paliativos. Esse tipo de cuidado é definido pela $\mathrm{OMS}^{5}$ como uma abordagem que objetiva a melhoria da qualidade de vida dos pacientes e seus familiares, por meio da prevenção e alívio do sofrimento, identificação precoce, avaliação e tratamento impecável da dor e de outros problemas de ordem física, psicossocial e espiritual.

Pacientes com câncer avançado em cuidados paliativos exclusivos tendem a apresentar menor sobrevida justificada pela carga de doença e suspensão do tratamento anticâncer ${ }^{6}$. Supõe-se que tal grupo de indivíduos, e infectados pela Covid-19, experimentará ainda maior reduçáo de sobrevida, o que torna necessário o apoio de uma equipe multiprofissional de paliativistas para alívio do sofrimento, controle dos sintomas, promoção da qualidade de vida e de morte ${ }^{7}$. Aprimorar a assistência a esses pacientes, oferecendo um cuidado integral e otimizando os recursos disponíveis, é um dos principais desafios para o Sistema Único de Saúde (SUS).

Dessa forma, destaca-se, neste documento, algumas orientações para a assistência de pacientes com câncer avançado acometidos pela Covid-19, com base em documentos publicados sobre a infecção, nos princípios e diretrizes dos cuidados paliativos e na experiência da Unidade de Cuidados Paliativos exclusivos do Hospital do Câncer IV (HC IV) do Instituto Nacional de Câncer José Alencar Gomes da Silva (INCA). O objetivo é que este manuscrito possa servir como mola propulsora para o desenvolvimento de estratégias de cuidado que aliem o controle de sintomas às questóes relativas à humanização da assistência, possibilitando que as demandas dessa população sejam atendidas, com consequente qualidade de vida e de morte.

\footnotetext{
${ }^{1}$ Especialista. Médica. Unidade de Cuidados Paliativos do Hospital do Câncer IV (HC IV) do Instituto Nacional de Câncer José Alencar Gomes da Silva (INCA). Rio de Janeiro (RJ), Brasil. Orcid iD: https://orcid.org/0000-0001-7285-8768

${ }^{2}$ Especialista. Enfermeira. Unidade de Cuidados Paliativos/HC IV/INCA. Rio de Janeiro (RJ), Brasil. Orcid iD: https://orcid.org/0000-0002-6430-1932

${ }^{3}$ Especialista. Nutricionista. Unidade de Cuidados Paliativos/HC IV/INCA. Rio de Janeiro (RJ), Brasil. Orcid iD: https://orcid.org/0000-0002-0951-8725

${ }^{4}$ Mestre. Enfermeira. Unidade de Cuidados Paliativos/HC IV/INCA. Rio de Janeiro (RJ), Brasil. Orcid iD: https://orcid.org/0000-0003-4608-0918

${ }^{5}$ Doutora. Médica. Unidade de Cuidados Paliativos/HC IV/INCA. Rio de Janeiro (RJ), Brasil. Orcid iD: https://orcid.org/0000-0001-5537-7399

${ }^{6}$ Especialista. Enfermeira. Unidade de Cuidados Paliativos/HC IV/INCA. Rio de Janeiro (RJ), Brasil. Orcid iD: https://orcid.org/0000-0002-4087-8585

${ }^{7}$ Mestre. Psicóloga. Unidade de Cuidados Paliativos/HC IV/INCA. Rio de Janeiro (RJ), Brasil. Orcid iD: https://orcid.org/0000-0003-4726-1016

${ }^{8}$ Especialista. Enfermeira. Unidade de Cuidados Paliativos/HC IV/INCA. Rio de Janeiro (RJ), Brasil. Orcid iD: https://orcid.org/0000-0002-8158-7637

${ }^{9}$ Especialista. Enfermeira. Unidade de Cuidados Paliativos/HC IV/INCA. Rio de Janeiro (RJ), Brasil. Orcid iD: https://orcid.org/0000-0003-4665-7545

${ }^{10}$ Doutora. Nutricionista. Unidade de Cuidados Paliativos/HC IV/INCA. Rio de Janeiro (RJ), Brasil. Orcid iD: https://orcid.org/0000-0002-5052-1846

Endereço para correspondência: Livia Costa de Oliveira. Rua Visconde de Santa Isabel, 274 - Vila Isabel. Rio de Janeiro (RJ), Brasil. CEP $20.560-120$.

E-mail: lillycostaoliveira@gmail.com
} 


\section{DESENVOLVIMENTO}

A situação atual demanda pela revisão de processos e protocolos assistenciais para a garantia da qualidade dos serviços e da segurança dos pacientes e profissionais das diversas unidades de saúde do país e no mundo ${ }^{8,9}$.

A OMS publicou um documento sugerindo a reorganização dos fluxos de funcionamento de serviços de saúde para lidar com pacientes suspeitos ou confirmados de infecção pela Covid-19, bem como seus contactantes ${ }^{7}$. Para a Agência Nacional de Vigilância Sanitária (Anvisa) ${ }^{10}$, os fluxos devem ser desenhados de maneira a atender às necessidades de cada realidade.

O Ministério da Saúde, em janeiro de 2020, instalou o Centro de Operações de Emergência em Saúde Pública Covid-19 (COE-Covid-19). Em março deste ano, foi instituído o Comitê de Gerenciamento de Crise do novo coronavírus para coordenar e monitorar as açóes internas relacionadas à doença no INCA.

Seguindo as orientaçóes dessas instâncias, integrantes da equipe multidisciplinar do HC IV elaboraram um plano denominado Planejamento Emergencial para Enfrentamento diante da Pandemia Causada pelo Sars- $\mathrm{CoV}-2$, visando à garantia da qualidade da assistência, proteçấo dos profissionais, pacientes e seus cuidadores/ familiares ${ }^{11}$.

Para melhor compreensão, cabe esclarecer que o HC IV conta com os seguintes setores assistenciais envolvidos neste manuscrito: Serviço de Pronto Atendimento (SPA) - destinado ao atendimento de urgências e emergências de pacientes matriculados na unidade; Ambulatório - destinado ao acompanhamento de pacientes com melhor performance status (KPS $>=50 \%$ ) e dos pacientes fora de critério para atendimento domiciliar; Assistência Domiciliar (AD) - destinada ao acompanhamento de pacientes com pior performance status $(\mathrm{KPS}<50 \%)$ que residam em um raio de até $80 \mathrm{~km}$ de distância do HC IV; Internaçáo Hospitalar (IH) - destinada ao manejo de sintomas agudizados e cuidados ao fim de vida.

A seguir, estão descritas algumas orientações para assistência ao paciente suspeito ou confirmado de infecçáo pela Covid-19, conforme os fluxos dos processos assistenciais do HC IV.

\section{ORIENTAÇÕES}

\section{SERVIÇO DE PRONTO ATENDIMENTO}

Os profissionais dos SPA deverão atender aos pacientes que procuram as unidades por livre demanda, seja presencialmente ou por ligação telefônica. Devem-se considerar as recomendaçóes para o uso de equipamentos de proteçáo individual (EPI) adequados nos atendimentos presenciais de pacientes suspeitos de infecção por Covid-197.

Todos os pacientes que demandem por atendimento presencial devem ser submetidos a uma pré-triagem de síndrome gripal/sintomas de Covid-19, antes de entrar no setor. A definiçấo de caso suspeito deverá se dar de acordo com os critérios estabelecidos pelas autoridades sanitárias. Pacientes com suspeita de infecção pela Covid-19 deverâo ser encaminhados para uma sala destinada exclusivamente a esse fim, recomendando-se a investigação clínica e epidemiológica individual. Após cada atendimento, a equipe assistencial deverá solicitar a limpeza e a desinfecção do ambiente.

Casos suspeitos de infecçáo pela Covid-19 com sintomas leves, que náo demandem por internação hospitalar, deverão ser liberados para residência com orientação de quarentena domiciliar por pelo menos 14 dias. O médico responsável pelo atendimento deverá fornecer atestado médico de igual período para o paciente e para todos os familiares que com ele residam (ainda que assintomáticos). Além disso, os Termos de Declaração e de Consentimento devem ser preenchidos, assinados e arquivados no prontuário, conforme Portaria n. ${ }^{\circ}$ 454, de 20 de março de 2020, do Ministério da Saúde ${ }^{12}$. Esses pacientes deverão ser sinalizados aos respectivos processos de origem para monitoramento remoto.

Casos suspeitos de infecção pela Covid-19, que demandem por internação hospitalar, deverão ser notificados em Ficha de Síndrome Respiratória Aguda Grave (SRAG), assim como deverá ser coletado swab de nasofaringe e orofaringe para pesquisa do Sars-CoV-2 pelo método de Transcrição Reversa seguida de Reação em Cadeia da Polimerase (do inglês, Reverse Transcription Polymerase Chain Reaction - RT-PCR). O paciente deverá ser encaminhado ao setor de Internaçáo Hospitalar o mais breve possível, reduzindo o tempo de permanência no SPA.

\section{ASSISTÊNCIA DOMICILIAR}

Todos os pacientes vinculados à $\mathrm{AD}$ deverão ser acompanhados por teleatendimento, por enfermeiro e/ ou médico, para monitoramento e controle dos sintomas relacionados à doença maligna avançada, assim como triar sintomas sugestivos de síndrome gripal/Covid-19. Em caso de paciente/familiar suspeito de infecção pela Covid-19, a equipe da $\mathrm{AD}$ não deverá ir a esse domicílio durante o período de quarentena (14 dias).

A Academia Nacional de Cuidados Paliativos $(\mathrm{ANCP})^{13}$ se manifestou favorável ao teleatendimento, sempre que possível. Essa forma de atendimento foi autorizada e normatizada pelos Conselhos Federais de Enfermagem (Resolução Cofen n. ${ }^{\circ}$ 634/2020) ${ }^{14}$, Medicina 
(Ofício CFM n.o $1.756 / 2020)^{15}$, Nutrição (Resolução CFN n. ${ }^{0}$ 646/2020) ${ }^{16}$, entre outros.

Por intermédio do teleatendimento, todos os profissionais da equipe multiprofissional devem avaliar, orientar e prescrever as açôes pertinentes à sua prática profissional, de maneira que o atendimento presencial fique destinado apenas aos pacientes que necessitem, desde que náo apresentem sintomas suspeitos de infecção pela Covid-19. Poderão ser feitos também ajustes das prescriçóes médicas e pedidos de material para cuidados em domicílio de acordo com a demanda identificada no atendimento a distância. Em caso de relato de piora dos sintomas, as famílias deverão ser orientadas a conduzirem os pacientes ao SPA.

\section{AMBULATÓRIO}

Todos os pacientes vinculados ao ambulatório deverão ser acompanhados por teleatendimento visando ao monitoramento e ao controle dos sintomas relacionados à doença maligna avançada, assim como triar sintomas sugestivos de síndrome gripal/Covid-19. Em caso de paciente/familiar suspeito de infecção pela Covid-19, estes não deverão comparecer a consultas presenciais de rotina no ambulatório pelo período de 14 dias, permanecendo em monitoramento remoto.

Por meio do teleatendimento, o enfermeiro e o médico deverão avaliar, orientar e prescrever as açôes pertinentes à sua prática profissional, de acordo com as normatizaçôes do CFM e do Cofen. Caso necessário, poderão ser solicitados orientaçôes/atendimentos telefônicos às demais categorias profissionais da equipe multi (psicologia, nutrição, fisioterapia e serviço social). O médico e/ou enfermeiro responsáveis pelo teleatendimento poderão deliberar sobre a necessidade de atendimento presencial.

\section{INTERNAÇÃO HOSPITALAR}

Em caso de indicação de internação hospitalar de paciente suspeito ou confirmado de infecção pela Covid-19, seus familiares deverão ser orientados sobre o quadro clínico pelo médico e dispensados ao domić́lio com orientaçáo de quarentena domiciliar de todos os contactantes. O local de internação será definido conforme a orientação da própria unidade em leitos exclusivos para esse fim. Além disso, o paciente poderá ser transferido para algum hospital de referência para tratamento da Covid-19, de acordo com o Plano de Resposta Estadual ao Coronavírus ${ }^{17}$. A regulação deverá ser realizada via Sistema Estadual de Regulação (SER).

$\mathrm{O}$ isolamento de pacientes com suspeita de infecção pela Covid-19 deve ser prioritário em relação aos outros motivos de isolamento. Os quartos deveráo ter a entrada sinalizada com um alerta referindo doença respiratória e terem acesso restrito aos trabalhadores da saúde envolvidos no atendimento.

Esse paciente não deverá ter acompanhante e nem receber visita. Portanto, boletins informativos do quadro clínico dos pacientes devem ser passados aos familiares referenciados por meio de telecomunicação. Visitas virtuais de familiares devem ser organizadas pela equipe, propiciando a humanização do ambiente durante o isolamento obrigatório, sem possibilidade de visitação presencial.

Em caso de suspeita de infecção pela Covid-19 em paciente já internado por causa oncológica, este deverá ser transferido para leito destinado a paciente suspeito e investigado com coleta de swab. Seu contactante de quarto deverá permanecer sob vigilância de sintomas respiratórios/ síndrome gripal em quarto não compartilhado, utilizando máscara cirúrgica, se tolerada. Em caso de RT-PCR detectável para Sars-CoV-2, o contactante também deverá ser transferido para leito de isolamento específico e ser submetido à coleta de swab.

\section{NOTIFICAÇÃO0}

O médico que suspeitar de infecção pela Covid-19 deverá preencher a ficha de registro individual para casos de síndrome gripal (casos leves liberados para domicílio) ou de SRAG-hospitalizado do Sistema de Informação de Vigilância Epidemiológica. Essas fichas deverão ser encaminhadas à Comissão de Controle de Infecção Hospitalar (CCIH) de cada unidade.

Caberá à CCIH enviar a ficha de SRAG por e-mail ao Serviço de Vigilância Epidemiológica da Área Programática 2.2 (AP 2.2), atualizar o(s) resultado(s) do(s) swab(s) coletado(s) e o desfecho do caso.

\section{Preparo do CORPO}

A Nota Técnica 04/2020 determina as diretrizes sobre o preparo e acondicionamento do corpo após o óbito de paciente suspeito/confirmado por infecção pelo novo coronavírus $^{18}$. Todos os profissionais de saúde e demais profissionais que terão contato com o cadáver deverão utilizar os EPI recomendados para Sars-CoV2.

$\mathrm{O}$ reconhecimento do corpo na morgue deverá acatar as recomendações da Anvisa: apenas um responsável, respeitando a distância de dois metros do corpo (garantida pela sinalização de faixa no chão).

O familiar/responsável receberá as orientações pertinentes pelo assistente social e/ou pelo médico que irá fornecer a Declaração de Óbito.

\section{CONCLUSÃO}

O formato da assistência prestada aos pacientes em cuidados paliativos oncológicos, em virtude da 
pandemia da Covid-19, precisou ser remodelado. No entanto, necessita-se manter foco sobre a promoção da qualidade de vida e de morte desses pacientes e de seus familiares, pautando-se sobre a comunicação e o trabalho em equipe multiprofissional. Dessa forma, busca-se, por meio deste manuscrito, auxiliar outros serviços de saúde a ofertarem um cuidado digno e de qualidade a pacientes com câncer avançado em cuidados paliativos, acometidos pela Covid-19.

\section{CONTRIBUIÇÕES}

Todos os autores contribuíram substancialmente na concepçáo e delineamento do estudo; obtenção, análise e interpretação dos dados; redação e revisão crítica e aprovaram a versão final a ser publicada.

\section{DECLARAÇÃO DE CONFLITO DE INTERESSES}

Nada a declarar.

\section{FONTES DE FINANCIAMENTO}

Não há.

\section{REFERÊNCIAS}

1. Fundaçáo Oswaldo Cruz. Processo de luto da COVID-19 [Internet]. Rio de Janeiro: FIOCRUZ; 2020 (Saúde mental e atenção psicossocial na pandemia COVID-19). [acesso 2020 maio 8]. Disponível em: http://www. fiocruzbrasilia.fiocruz.br/wp-content/uploads/2020/04/ Sa\%C3\%BAde-Mental-e-Aten\%C3\%A7\%C3\%A3oPsicossocial-na-Pandemia-Covid-19-processo-de-lutono-contexto-da-Covid-19.pdf

2. Merino MTGB. Palliative care: taking the long view. Front Pharmacol. 2018;9:1140. doi: https://doi. org/10.3389/fphar.2018.01140

3. Oh WK. COVID-19 infection in cancer patients: early observations and unanswered questions. Ann Oncol. 2020;31(7):838-839. doi: https://doi.org/10.1016/j. annonc.2020.03.297

4. Thuler LCS, Melo AC. Sars-CoV-2/Covid-19 em pacientes com câncer. Rev Bras Cancerol. 2020;66(2):e00970. doi: https://doi.org/10.32635/2176-9745. RBC.2020v66n2.970

5. World Health Organization [Internet]. Geneva: WHO; c2020. Palliative care; 2018 Feb 19 [cited 2020 May 7]. Available from: https://www.who.int/en/news-room/ fact-sheets/detail/palliative-care

6. Silva GA, Wiegert EVM, Calixto-Lima L, et al. Clinical utility of the modified Glasgow Prognostic Score to classify cachexia in patients with advanced cancer in palliative care. Clin Nutr. 2020;39(5):1587-92. doi: https://doi.org/10.1016/j.clnu.2019.07.002

7. World Health Organization. Home care for patients with suspected novel coronavirus $(\mathrm{nCoV})$ infection presenting with mild symptoms and management of contacts: interim guidance [Internet]. Geneva: WHO; 2020 Jan 20 [cited 2020 May 05]. Available from: https://apps.who.int/iris/ bitstream/handle/10665/330671/9789240000834-eng. pdf?sequence $=1$ \&isAllowed $=y$

8. Yang G, Zhang H, Yang Y. Challenges and countermeasures of integrative cancer therapy in the epidemic of COVID-19. Integr Cancer Ther. 2020;19:1534735420912811. doi: https://doi. org/10.1177/1534735420912811

9. Piovacari SMF, Santos GFCG, Santana GA, et al. Fluxo de assistência nutricional para pacientes admitidos com COVID-19 e SCOVID-19 em unidade hospitalar. BRASPEN J [Internet]. 2020 [acesso 2020 maio 18];35(1):6-8. Disponível em: https://66b28c71-9a364ddb-9739-12f146d519be.usrfiles.com/ugd/66b28c_2 f5d298499184d22b2655dff908f58c9.pdf

10. Agência Nacional de Vigilância Sanitária. Nota Técnica GVIMS/ GGTES/ ANVISA No 04/2020: orientaçôes para serviços de saúde: medidas de prevenção e controle que devem ser adotadas durante a assistência aos casos suspeitos ou confirmados de infecção pelo novo coronavírus (SARS-CoV-2) [Internet]. Brasília, DF: ANVISA; 2020 jan 30 [atualizada 2020 maio 8; acesso 2020 maio 15]. Disponível em: http:// portal.anvisa.gov.br/documents/33852/271858/ Nota+T\%C3\%A9cnica+n+04-2020+GVIMS-GGTESANVISA/ab598660-3de4-4f14-8e6f-b9341c196b28

11. Instituto Nacional de Câncer José Alencar Gomes da Silva. Hospital do Câncer IV: planejamento emergencial para enfrentamento diante da pandemia causada pelo SARS-CoV-2 (COVID -19). Rio de Janeiro: INCA; 2020 abr.

12. Ministério da Saúde (BR). Portaria no 454, de 20 de março de 2020. Declara, em todo território nacional, o estado de transmissão comunitária do coronavírus (COVID-19). Diário Oficial da União, Brasília, DF; 2020 mar 20. Seção 1-Extra, p. 1.

13. Crispim D, Santos AFJ. Assistência domiciliar para pacientes em acompanhamento em cuidados paliativos durante a pandemia COVID-19 [Internet]. São Paulo: ANCP; [2020] [acesso 2020 maio 14]. Disponível em: https://www.dropbox.com/s/2iccllm3yapka1j/03_ ANCP_Ebook_Assist\%C3\%AAncia\%20Domicilia_ Acompanhamento_Cuidados_Paliativos_COVID-19. pdf?dl=0 /.

14. Conselho Federal de Enfermagem (BR). Resolução no 634, de 26 de março de 2020. Autoriza e normatiza, "ad referendum" do Plenário do Cofen, a teleconsulta de enfermagem como forma de combate à pandemia provocada pelo novo coronavírus (Sars-Cov-2), 
mediante consultas, esclarecimentos, encaminhamentos e orientaçóes com uso de meios tecnológicos, e dá outras providências [Internet]. Diário Oficial da União, Brasília, DF; 2020 mar 27. Seçấo 1, p. 117 [acesso 2020 maio 10]. Disponível em: http://www.cofen.gov.br/wp-content/ uploads/2020/03/Resolu\%C3\%A7\%C3\%A3o-Cofenn\%C2\%BA-634-2020.pdf

15. Conselho Federal de Medicina (BR). Ofício CFM n ${ }^{\circ}$ 1756/2020 - COJUR [Internet]. Brasília, DF: CFM; 2020 mar 19. Assunto: Telemedicina [acesso 2020 maio 10]. Disponível em: http://portal.cfm.org.br/images/ PDF/2020_oficio_telemedicina.pdf

16. Conselho Federal de Nutricionistas (BR). Resolução CFN nº646, de 18 de março de 2020. Suspende até o dia 31 de agosto de 2020 o disposto no artigo 36 da Resolução CFN no 599, de 25 de fevereiro de 2018, que aprova o Código de Ética e de Conduta dos Nutricionistas [Internet]. Diário Oficial da União, Brasília, DF; 2020 mar 19. Seçấo I, p. 81 [acesso 2020 maio 10]. Disponível em: http://www.in.gov.br/en/web/dou/-/resolucao-n646-de-18-de-marco-de-2020-248808850

17. Secretaria de Estado de Saúde (RJ). Plano de resposta de emergência ao coronavírus no Estado do Rio de Janeiro [Internet]. Rio de Janeiro: Secretaria de Estado de Saúde; 2020 jan 31 [acesso 2020 maio 16]. Disponível em: https://www.saude.gov.br/images/pdf/2020/ fevereiro/13/PLANO-DE-CONTINGENCIA-novocoronavirus-RIO-DE-JANEIRO-EM-REVIS--O.pdf

18. Ministério da Saúde (BR). Manejo de corpos no contexto do novo coronavírus COVID-19 [Internet]. Versão 1. Brasília, DF: Ministério da Saúde; 2020 mar 25 [acesso 2020 abr 10]. Disponível em: https://www.saude.gov.br/ images/pdf/2020/marco/25/manejo-corpos-coronavirusversao1-25mar20-rev5.pdf 